\title{
Holographic Dark Energy Cosmological Model in Scalar Tensor Theory of Gravitation
}

\author{
V.G.Mete, ${ }^{1}$ V.D.Elkar, ${ }^{2}$ Poonam P. Kadu \\ Department of Mathematics, R.D.I.K. \& K.D. College, Badnera-Amravati, India. \\ 1,2Department of Mathematics, J.D.Patil Sangludkar Mahavidyalaya, Daryapur, Dist.Amravati \\ Email: vmete5622@gmail.com
}

\begin{abstract}
An attempt has been taken to study the minimally interacting fields, dark matter and holographic dark energy in scalar tensor theory formulated by Saez and Ballester. The exact solution of the field equations is obtained by using the fact that the scalar expansion of the universe is proportional to the shear scalar. Some physical and geometrical aspects of the model are also discussed.
\end{abstract}

Keywords: Bianchi type VIo universe, scalar- tensor theory, holographic dark energy.

\section{Introduction}

Various observational data of modern cosmology based on measurement indicate that our universe is in the process of an accelerated expansion. This was observed by Reiss et al.[1], SNeIa (Perlmutter et al.[2]), WMAP (Bennett[3]), SDSS(Tegmark[4]) and X-ray (Allen[5]). The concept of dark energy refers to a kind of exotic energy with negative pressure whose origin skill remains a mystery. Astronomical observation i.e. Wilkinson, Microwave Astrophysics Probe indicates that the dark energy occupies $76 \%$ of the energy of our universe. $20 \%$ dark matter and baryonic matter and radiations occupy about $4 \%$ of total energy of the universe. The nature of the dark energy and dark matter is unknown and many radically different models have been proposed such as at tiny positive cosmological constant, quintessence (Caldwell; et al [6],Liddle and Scherrer [7], Steinhardt et al. [8], Dvali et al.[9], Deffayet [10]); the nonlinear E(R) models (Capozziello et al. [11], Carroll et al. [12], Nojiri and Odintsov [13]) and dark energy in brine worlds.

Einstein himself pointed out that general relativity does not account satisfactorily for inertial properties of matter, i.e. Mach's principle is not substantiated by general relativity. So in recent years, there has been a lot of interest in several alternative theories of gravitation. The most important among them are scalar tensor theories of gravitation formulated by Brans and Dicke ([14], Nordtvedt [15] and Saez and Ballester [16]). All versions of the scalar- tensor theories are based on the introduction of a scalar field $\varphi$ into the formulation of general relativity, this scalar field together with the metric tensor field then forms a scalar tensor field representing the gravitational field.

In Saez-Ballester theory, the metric is coupled with a dimensionless scalar field in a simple manner. This coupling gives a satisfactory description of weak fields and suggests a possible way to solve missing matter problem in non-flat FRW cosmologies.

The Saez-Ballester [16] field equations are

$$
G_{i j}-\omega \varphi^{n}\left(\varphi_{, i} \varphi_{, j}-\frac{1}{2} g_{i j} \varphi_{, k} \varphi^{, k}\right)=-\left(T_{i j}+\bar{T}_{i j}\right),
$$

The scalar field $\varphi$ satisfies the following equation

$$
2 \varphi^{n} \varphi_{, i}^{i}+n \varphi^{n-1} \varphi_{, k} \varphi^{k}=0
$$

where $G_{i j}=R_{i j}-\frac{1}{2} R g_{i j}$ is the Einstein tensor, $T_{i j}$ and $\bar{T}_{i j}$ are energy momentum tensor of matter and holographic dark energy, $\omega$ and $n$ are constants , comma (,) and semicolon (;) denotes partial and covariant differentiation respectively. 
A detailed discussion of Saez-Ballester cosmological models is contained in the work of Saez[16], Singh and Agrawal[17], Shri Ram and Tiwari[18], Recently Adhav et al. [19] have studied Axially symmetric non-static domain walls in scalar-tensor theories formulated by Brans and Dick [14] and SaezBallester[16].

Using holographic principal of quantum gravity theory (Susskind [20]) a viable holographic dark energy model was constructed by Li [21]. The holographic dark energy model is successful in explaining the observational data. The several authors, in particular, Sarkar and Mahanta [22] studied the evolution of holographic dark energy in Bianchi type -I space time with constant deceleration parameter. Recently Sarkar [23] studied holographic dark energy model in Bianchi type -I space time with linearly varying deceleration parameter and established a corresponding with generalized Chapligin gas models of the universe. Setare [24] studied holographic dark energy model in Brans-Dicke theory. Sarkar [25, 26, 27] has investigated Bianchi type-II cosmological model with interacting holographic dark energy. Very recently Setare and Vanegas [28] have investigated the cosmological dynamics of interacting holographic dark energy model. Kiran et al. [29] have studied Bianchi type- V minimally interacting holographic dark energy model in the scalar- tensor theory of gravitation proposed by Saez and Ballester. Adhav et al. [30] have investigated plane symmetric space- time with interacting dark matter and holographic dark energy exponential volumetric expansion. Reddy at al. [31] have discussed Kaluza-Klein minimally interacting holographic dark energy model in scalar tensor theory of gravitation. Recently Rao, V.U.M.et al. [32] have constructed Kantowski-Sachs holographic dark energy in Brans-Dicke theory of gravitation. Motivated with these works, we have studied in this paper, minimally interacting fields dark matter and holographic dark energy in Saez and Ballester scalar tensor theory of gravitation. Our paper is organized as follows.

In section 2, we derive the field equations. In section 3, we deal with the solution of the field equations in presence of holographic dark energy and dark matter. Section 4 is mainly concerned with the physical and kinematical properties. The last section is the conclusion.

\section{$2 \quad$ Metric and Field Equations}

We consider the Bianchi Type $\mathrm{VI}_{0}$ metric in the form

$$
d s^{2}=-d t^{2}+A^{2}(t) d x^{2}+B^{2}(t) \exp (-2 q x) d y^{2}+C^{2}(t) \exp (2 q x) d z^{2}
$$

where $q$ is nonzero constant and $A, B, C$ are scale functions of $t$ only.

The energy momentum tensor of matter and holographic dark energy are defined as

$$
\begin{gathered}
T_{i j}=\rho_{m} u_{i} u_{j} \\
\bar{T}_{i j}=\left(\rho_{\lambda}+p_{\lambda}\right) u_{i} u_{j}+p_{\lambda} g_{i j}
\end{gathered}
$$

where $\rho_{m}, p_{\lambda}$ are the energy densities of matter and holographic dark energy and $p_{\lambda}$ is the pressure of the holographic dark energy.

The Saez-Ballester field equations (1) and (2) for the metric (3) with the help of equations (4)-(5) can be written as

$$
\begin{gathered}
\frac{\ddot{B}}{B}+\frac{\ddot{C}}{C}+\frac{\dot{B} \dot{C}}{B C}+\frac{q^{2}}{A^{2}}+\frac{\omega}{2} \varphi^{n} \dot{\varphi}^{2}=-p_{\lambda} \\
\frac{\ddot{A}}{A}+\frac{\ddot{C}}{C}+\frac{\dot{A} \dot{C}}{A C}-\frac{q^{2}}{A^{2}}+\frac{\omega}{2} \varphi^{n} \dot{\varphi}^{2}=-p_{\lambda} \\
\frac{\ddot{B}}{B}+\frac{\ddot{A}}{A}+\frac{\dot{A} \dot{B}}{A B}-\frac{q^{2}}{A^{2}}+\frac{\omega}{2} \varphi^{n} \dot{\varphi}^{2}=-p_{\lambda} \\
\frac{\dot{A} \dot{B}}{A B}+\frac{\dot{A} \dot{C}}{A C}+\frac{\dot{B} \dot{C}}{B C}-\frac{q^{2}}{A^{2}}-\frac{\omega}{2} \varphi^{n} \dot{\varphi}^{2}=\rho_{m}+\rho_{\lambda} \\
\frac{\dot{B}}{B}-\frac{\dot{C}}{C}=0
\end{gathered}
$$




$$
\ddot{\varphi}+\left(\frac{\dot{A}}{A}+\frac{\dot{B}}{B}+\frac{\dot{C}}{C}\right) \dot{\varphi}+\frac{n}{2} \frac{\dot{\varphi}^{2}}{\varphi}=0
$$

where (.) over the symbols $A, B, C$ denotes ordinary differentiation with respect to $t$.

The continuity equation of the matter and dark energy is given by

$$
\dot{\rho}_{m}+\rho_{m}(3 H)+\dot{\rho}_{\lambda}+3 H\left(1+w_{\lambda}\right) \rho_{\lambda}
$$

We assume that the matter and holographic dark energy interact with each other hence both the components conserve separately, so that

The continuity equation of holographic dark energy is

$$
\dot{\rho}_{\lambda}+3 H\left(1+w_{\lambda}\right) \rho_{\lambda}=0
$$

and the continuity equation of matter is

$$
\dot{\rho}_{m}+3 H \rho_{m}=0
$$

where $w_{\lambda}=\frac{p_{\lambda}}{\rho_{\lambda}}$ is the barotropic equation of state parameter for holographic dark energy.

The physical and geometrical parameters for the space -time (3), we have the following forms

The average scale factor

$$
R=(A B C)^{\frac{1}{3}}
$$

The mean Hubble's parameter

$$
H=\frac{\dot{R}}{R}
$$

an important observed quantity in cosmology is the deceleration parameter $q$ which is defined as

$$
q=-\frac{R \ddot{R}}{\dot{R}^{2}}=-1-\frac{\dot{H}}{H}
$$

The average anisotropic parameter $\Delta$ is defined as

$$
\Delta=\frac{1}{3} \sum_{i=1}^{3}\left(\frac{\Delta H_{i}}{H}\right)^{2}
$$

where $\Delta H_{i}=H_{i}-H$ and $H_{i}(i=1,2,3)$ represent the directional Hubble parameters, the shear scalar expansion $(\theta)$, and the shear scalar $(\sigma)$ are

$$
\begin{gathered}
\theta=\frac{\dot{A}}{A}+\frac{\dot{B}}{B}+\frac{\dot{C}}{C} \\
\sigma^{2}=\frac{1}{2}\left(\frac{\dot{A}^{2}}{A^{2}}+\frac{\dot{B}^{2}}{B^{2}}+\frac{\dot{C}^{2}}{C^{2}}-\frac{\dot{A} \dot{B}}{A B}-\frac{\dot{B} \dot{C}}{B C}-\frac{\dot{A} \dot{C}}{A C}\right)
\end{gathered}
$$

\section{Solution to the Field Equations}

Equation (10) leads to

$$
B=k C
$$

where $k$ is integrating constant.

Using the equation (20), the system of field equations (6)-(11) reduces to

$$
\begin{aligned}
& 2 \frac{\ddot{C}}{C}+\left(\frac{\dot{C}}{C}\right)^{2}+\frac{q^{2}}{A^{2}}+\frac{\omega}{2} \varphi^{n} \dot{\varphi}^{2}=-p_{\lambda} \\
& \frac{\ddot{A}}{A}+\frac{\ddot{C}}{C}+\frac{\dot{A} \dot{C}}{A C}-\frac{q^{2}}{A^{2}}+\frac{\omega}{2} \varphi^{n} \dot{\varphi}^{2}=-p_{\lambda}
\end{aligned}
$$




$$
\begin{gathered}
2 \frac{\dot{A} \dot{C}}{A C}+\left(\frac{\dot{C}}{C}\right)^{2}-\frac{q^{2}}{A^{2}}-\frac{\omega}{2} \varphi^{n} \dot{\varphi}^{2}=\rho_{m}+\rho_{\lambda} \\
\frac{\ddot{\varphi}}{\varphi}+\left(\frac{\dot{A}}{A}+\frac{\dot{B}}{B}+\frac{\dot{C}}{C}\right) \frac{\dot{\varphi}}{\varphi}+\frac{n}{2} \frac{\dot{\varphi}^{2}}{\varphi^{2}}=0
\end{gathered}
$$

The field equations (21)-(24) are the system of four equations with six unknowns $A, C, \rho_{m}, \rho_{\lambda}, p_{\lambda}, \varphi$ hence we need additional constraints to obtained solution of the system. We use the scalar expansion $\theta$ that is proportional to the shear scalar $\sigma^{2}$. So that we have (Collins et al. [33])

$$
A=C^{n}
$$

using the equations (21), (22) and (25) we obtain,

$$
\frac{\ddot{C}}{C}+(1+n)\left(\frac{\dot{C}}{C}\right)^{2}+\frac{2 q^{2}}{(1-n) C^{2 n}}=0
$$

we assume

$$
\begin{gathered}
\dot{C}=f(C) \\
\ddot{C}=f f^{\prime}
\end{gathered}
$$

where

$$
f^{\prime}=\frac{d f}{d C}
$$

equating (26), (27) and (28), we have

$$
\frac{d f^{2}}{d C}+2(1+n) \frac{f^{2}(C)}{C}=\frac{2 q^{2}}{(n-1) C^{2 n-1}}
$$

equation (29) yields

$$
f^{2}(C)=\frac{q^{2}}{2(n-1)} C^{2(1-n)}+\frac{\beta}{C^{2(1+n)}}
$$

where $\beta$ is an integrating constant.

Using the suitable choice of transformation i.e. $C=T, x=X . y=Y, z=Z$, the metric (3) reduces to the form

$$
d s^{2}=-\left[\frac{q^{2}}{2(n-1)} T^{2(1-n)}+\frac{\beta}{T^{2(1+n)}}\right]^{-1} d T^{2}+T^{2 n} d X^{2}+(k T)^{2} \exp (-2 q X) d Y^{2}
$$

The scalar field $\varphi$ in the model is

$$
\varphi^{\frac{n+2}{2}}=N \varphi_{0} \sqrt{\frac{q^{2}}{2(n-1) T^{4 n}}+\frac{\beta}{T^{4(1+n)}}}
$$

where the constant $\psi_{0}$ is omitted and

$$
N=\frac{-(n+2)}{2(n+1)}
$$

The pressure of holographic dark energy $p_{\lambda}$ of model (32) is

$$
p_{\lambda}=\frac{-q^{2}}{2(n-1) T^{2 n}}+\frac{\beta(2 n+1)+\omega / 2 \varphi_{0}^{2}}{T^{2(n+2)}}
$$

using equation (14), we get the energy density $\rho_{m}$ of dark matter

$$
\rho_{m}=\frac{\rho_{0}}{T^{n+2}}
$$

where $\rho_{0}$ is integrating constant and energy density $\rho_{\lambda}$ of holographic dark energy is 


$$
\rho_{\lambda}=\frac{2 q^{2}}{(n-1) T^{2 n}}+\frac{2 \beta(n+1)-\omega / 2 \varphi_{0}^{2}}{T^{2(n+2)}}-\frac{\rho_{0}}{T^{n+2}}
$$

The equation of state parameter $w_{\lambda}$ of holographic dark energy is

$$
w_{\lambda}=\left\{\frac{\left(\frac{-q^{2}}{2(n-1) T^{2 n}}+\frac{\beta(2 n+1)+\omega / 2}{T^{2(n+2)}}\right)}{\left(\frac{2 q^{2}}{(n-1) T^{2 n}}+\frac{2 \beta(n+1)-\omega / 2 \varphi_{0}^{2}}{T^{2(n+2)}}-\frac{\rho_{0}}{T^{n+2}}\right)}\right\}
$$

which shows that $w_{\lambda}$ is a function of time.

The coincident $r=\frac{\rho_{\lambda}}{\rho_{m}}$ i.e. the ratio of dark matter energy density to the dark energy density is given by

$$
r=\frac{\frac{2 q^{2}}{T^{n-2}}+\frac{2 \beta(n+1)-\omega / 2}{T_{0}^{2}}-\rho_{0}}{\rho_{0}}
$$

The matter density parameter $\Omega_{m}$ and holographic dark energy density parameter $\Omega_{\lambda}$ are given by

$$
\Omega_{m}=\frac{\rho_{m}}{3 H^{2}} \text { and } \Omega_{\lambda}=\frac{\rho_{\lambda}}{3 H^{2}}
$$

From equations (23), (30), (33) and (39), we obtain the overall density i.e. the sum of density parameter as

$$
\Omega_{m}+\Omega_{\lambda}=\frac{3}{(n+2)^{2}}\left[(2 n+1)-\frac{q^{2}}{\left(\frac{q^{2}}{2 n+1}+\frac{\beta}{T^{4}}\right)}-\frac{\omega / 2 \varphi_{0}^{2}}{\left(\frac{q^{2} T^{4}}{2(n-1)}+\beta\right)}\right]
$$

Here we observe that the sum of energy density parameter approaches to constant at the late time, so at late time the universe becomes flat.

\section{Some Physical and Kinematical Properties of the Model}

Some physical and kinematical parameters of cosmological model have the following expression

Spatial volume

$$
V=k T^{n+2}
$$

Scalar expansion

$$
\theta=(n+2) \sqrt{\frac{q^{2}}{2(n-1) T^{2 n}}+\frac{\beta}{T^{2(n+2)}}}
$$

Shear scalar

$$
\sigma^{2}=\frac{(n-1)^{2}}{3}\left(\frac{q^{2}}{2(n-1) T^{2 n}}+\frac{\beta}{T^{2(n+2)}}\right)
$$

Hubble parameter

$$
H=\frac{n+2}{3} \sqrt{\frac{q^{2}}{2(n-1) T^{2 n}}+\frac{\beta}{T^{2(n+2)}}}
$$


Anisotropic parameter

$$
\bar{A}=\frac{4}{3}
$$

\section{Conclusion}

In this paper, we have investigated the Bianchi type- $\mathrm{VI}_{0}$ cosmological model filled with dark matter and holographic dark energy in scalar- tensor theory of gravitation proposed by Saez and Ballester [16]. The exact solution of the field equations is obtained by using the fact that the scalar expansion of the universe is proportional to the shear scalar. It is observed that the holographic dark energy density decreases with the evolution of the universe and the average density parameter approaches to constant at late time, so the universe becomes flat which is supported to recent observations. We observe that the spatial volume $V$ is zero at $T=0$. At this epoch, the physical parameters $H, \theta, \sigma, \rho_{m}, \rho_{\lambda}$ and $p_{\lambda}$ are all infinite. Therefore the model has a point singularity at $T=0$. As time increases, the spatial volume increases and the physical parameters decrease and ultimately tend to zero as $T \rightarrow \infty$.

Therefore the ratio $\frac{\sigma}{\theta}$ does not tend to zero as $T \rightarrow \infty$. Therefore the shear scalar does not tend to zero faster than the expansion at late time.

\section{References}

1. A. G. Riess et al., Astron. J. 116, 1009(astro-ph/9805201), 1998.

2. S. Perlmutter et al., Astrophys. J. 517, 5 (astro-ph/9812133), 1999.

3. C.L. Bennet et al., Astrophys. J. Suppl. Ser. 148, 1, 2003.

4. M. Tegmark et al., (SDSS collaboration) Astrophys. J. Suppl. 69, 103501, 2004.

5. S.W. Allenet al., Mon. Not. R. Astron Soc. 353, 457, 2007.

6. R. Cadwell, R. Dave andP.J. Steinhardt, Phys. Rev.Lett. 80, 1582, 1998.

7. A.R. Liddel and R.J.Scherrer, Phys. Rev.D59.023509, 1999.

8. P.J. Steinhardt, L. Wang and I. Zlatev, Phys.Rev.D59, 123504, 1999.

9. G.R., Dvali, G. Gabadadze and M. Porrati,M., Phys. Lett. B 484, 112, 2000.

10.C. Deffayet, Phys. Lett. B 502, 199, 2001.

11.S. Capozziello, Carloni and A.Troisi, arXiv: astro-ph/0303041, 2003.

12.S.M. Carrol, et al., Phys. Rev. D 70, 043528, 2003.

13.S. Nojiri and S.D.Odintsov, Phys.Rev.D 68, 123512, 2003.

14.C.H. Brans and R. H. Dicke, Phys. Rev.124156, 925157 6,1961.

15.K. Nordtvedt, Post-Newtonian Metric for a General Class of Scalar-Tensor Gravitational Theories and Observational Consequences.; Ap. J. 161163, 1059, 1970.

16.D. Saez and V.J.Ballester, Phys. Lett.A 113168, 467, 1985.

17.T. Singh and A.K.Agrawal, Astrophys.Space Sci. 182, 289, 1991.

18.Shri Ram and S.K.Tiwari, Astrophys.Space Sci. 277, 461, 170, 1998.

19.K.S.Adhav, A.S. Nimkar and R.L. Naidu, Astrophys, Space Sci, 312, 165-169, 2007.

20.L.Susskind, J.Math.Phys.36, 6377, 1995.

21.M.Li, Phys.Lett.B, 603, 1, 2004.

22.S. Sarkar and C.R.Mahanta, Int.J.Theor.Phys. 52, 1482, 2013.

23.S. Sarkar, Astrophys. Space Sci. 349, 985, 2014.

24.M.R.Setare, Phs.Lett. B, 644, 99, 2007.

25.S. Sarkar, Astrophysics and Space Science, 349(2), 985, 2014.

26.S. Sarkar, Astrophysics and Space Science,DOI10.1007/s10509-014-1920-0, 2014.

27.S.Sarkar, Astrophysics and Space Science: 350(2), 821, 2014.

28.M.R.Setare and E.C.Vanegas, Int.J.Mod.Phys.D18, 147, 2009.

29.M.Kiran et al., Astrophys.Space Sci. DOI 10.1007/s10509-014-2099-o. 
30.K.S. Adhav, V.B.Raut and D.K. Joshi,Eur.J.Sci. and Tech 3,9, 2014.

31.D.R.K. Reddy and V.D. Elkar,Prespacetime J. 6,4 295-304, 2015.

32.V.U.M. Rao and U.Y.Divya Prasanthi, The African Reviews of Physics, 11, 001, 2016.

33.B.Collins et al., Gen. Relativ.Gravit. 12, 805, 1980. 